\title{
Testing of the transionospheric radiochannel using data from the global GPS network
}

\author{
Edward L. Afraimovich and Vitaliy A. Karachenschev \\ Institute of Solar-Terrestrial Physics, Russian Academy of Sciences, Irkutsk, Russia
}

\begin{abstract}
Using the international ground-based network of two-frequency receivers of the GPS navigation system provides a means of carrying out a global, continuous and fully-computerized monitoring of phase fluctuations of signals from satellite-borne radio engineering systems caused by the Earth's inhomogeneous and nonstationary ionosphere. We found that during major geomagnetic storms, the errors of determination of the range, frequency Doppler shift and angles of arrival of transionospheric radio signals exceeds that for magnetically quiet days by one order of magnitude as a minimum. This can be the cause of performance degradation of current satellite radio engineering navigation, communication and radar systems as well as superlong-baseline radio interferometry systems.
\end{abstract}

Key words total electron content - GPS - transionospheric radiochannel - geomagnetic disturbances - space weather

\section{Introduction}

With the development of progress, our civilization is becoming increasingly dependent on technological navigation and radar systems whose performance is to a certain extent governed by geospace conditions. In an effort to pave the way for tackling the issues of vulnerability of technological systems, the US National «Space Weather» program was developed (http://www.ofcm.gov/nswp-ip/text/cover.htm).

Radio Engineering Satellite Systems (RESS), with their ground-based and space-borne support facilities, are finding ever-widening application in various spheres of human activity. They are able to

Mailing address: Prof. Edward L. Afraimovich, Institute of Solar-Terrestrial Physics, Russian Academy of Science, P.O. Box 4026, Irkutsk, 664033, Russia; e-mail: afra@iszf.irk.ru provide global coverage, accuracy, continuity, high reliability and meet a number of other requirements imposed when tackling a broad spectrum of engineering problems. However, the use of RESS also implies new (and, in some cases, more stringent) requirements dictated by the need to ensure safety and economic efficiency of the operation of ground-based and airborne facilities, and to solve special problems (observation, aerophotography, searching and rescue of distressed transport vehicles and people). This applies equally for performance of Global Navigation Satellite Systems (GNSS) and for very-longbaseline radio interferometers (VLBI) (Thompson et al., 1986).

One of the most dramatic examples of the GNSS-GPS (Hofmann-Wellenhof et al., 1992) that has embodied many modern achievements at the interface of many sciences and technologies, has currently become a powerful factor of worldwide scientific and technical progress and is widely used in quite various realms of human activity. In this connection, much attention is given to continuous perfection of the GPS system and to the widening of the scope of its application for solving navigation problems them- 
selves, as well as for developing higher-precision systems for time and accuracy determinations. Even greater capabilities are expected in the near future through the combined use of the GPS with a similar Russian system GLONASS (Kharisov et al., 1998) and EU Galileo GNSS (Gibbons, 2002).

The broad prospects afforded by the use of positioning systems through the use of the GNSS dictate the need for a detailed study of the parameters of the satellite navigation systems themselves, including the reliability of their operation and noise immunity, especially when operated in extreme conditions (for instance, during large geomagnetic disturbances). To carry out such investigations requires considerable hardware, software and financial expenses incurred by the setting up of the necessary testing grounds for large sets of GPS-GLONASS receivers of different types, and for the development of dedicated software-hardware capabilities and facilities for data processing.

Meanwhile a global ground-based network of two-frequency multichannel GPS receivers is currently in operation, the data from which with a temporal resolution of $30 \mathrm{~s}$ are posted in a centralized fashion on the SOPAC server (ftp://lox.ucsd.edu) in a standard RINEX format (Gurtner, 1993). That part of this network that provides data which are publicly available at the beginning of 2002 consisted of more than 1000 sites. Posting of the data on the Internet opens up new avenues for a global, continuous, fully computerized monitoring of phase fluctuations of signals and associated errors of RESS performance. The database, obtained in this way, represents unique material.

Regional dedicated GPS receivers have been deployed to date to solve a variety of problems in geodynamics, to investigate the ionosphere, and for other purposes. The most dense GEONET network consisting of 1000 receivers has been in operation in Japan for over 5 years now (Saito et al., 2001, 2002).

Using multichannel GPS receivers at two coherently-coupled frequencies $f_{1}=1575.42$ $\mathrm{MHz}$ and $f_{2}=1227.60 \mathrm{MHz}$, highly accurate measurements of the group and phase delays are underway along the Line Of Sight (LOS) between the receiver on the ground and the transmitters on-board the GPS satellites which are in the zone of reception.

These data, converted to values of Total Electron Content (TEC), are of considerable current use in the study of the regular ionosphere and of disturbances of natural and technogenic origins (solar eclipses, flares, earthquakes, volcanoes, strong thunderstorms, auroral heating, nuclear explosions, chemical explosion events, launches of rockets). We do not cite here the relevant references for reasons of space, which account for hundreds of publications to date.

These prospects make the use of the global GPS network attractive for the implementation of the above-mentioned research, for preliminary accumulation of the sample statistics, as well as for carrying out analyses and modelings with the purpose of studying the GPSGLONASS. All this would make it possible to optimize the expenditure incurred by the creation of research grounds and by tests, as well as to obtain preliminary results virtually without any expenses for acquisition of equipment.

Recently a number of authors (Wilson et al., 1995; Mannucci et al., 1998; Schaer et al., 1998) have developed a new technology for constructing Global Ionospheric Maps (GIM) of TEC using IONEX data from the international IGS-GPS network. The GIM technology and its uses have been reported in a large number of publications (Wilson et al., 1995; Mannucci et al., 1998).

Two-hour TEC maps are readily accessible to any user, calculated by several research groups in the USA and Europe and are availabe on the Internet in the standard IONEX format (ftp://cddisa.gsfc.nasa.gov/pub/gps/products/ionex). It is also possible to obtain 15 min maps if necessary.

The ideology and automated software complex GLOBDET for global GPS detection and monitoring of ionospheric disturbances has been developed at the ISTP SD RAS. GLOBDET makes it possible to automate the acquisition, filtering and pretreatment process of the GPS data received via the Internet (Afraimovich, 2000). The global GPS detector is distinguished from previously available ionospheric radio sounding facilities by the continuity of observations, high spatial and temporal resolution and high sensitivity, as well as by standardization and adaptability of data processing. 
The global GPS detector can be used as a tester of the transionospheric radio channel of propagation of signals from space-based RESS and space radio sources.

The objective of this paper is to demonstrate - on the basis of using the GIM technology (Section 4) and GLOBDET technology (Sections 5 and 6) - how ionospheric disturbances during magnetic storms contribute to the signal degradation of radio engineering satellite systems.

\section{Influence of the ionosphere on transiono- spheric radio signal characteristics}

The performance of modern global satellite radio navigation systems that utilize the "EarthSpace» radio wave propagation channel is limited considerably by the influence of the geospace environment. Furthermore, the main contribution comes from systematic ionospheric effects of radio wave propagation: the group and phase delay, the frequency Doppler shift, and the rotation of the plane of polarization (Faraday effect). In many instances, the degree of manifestation of the above effects has only a weak dependence on the local distribution of electronic density in the ionosphere but is directly correlated with the value of TEC along the radio signal propagation path (Goodman and Aarons, 1990).

The key characteristic of the ionosphere that determines the variation of radio wave parameters is the TEC $I(t)$ or its derivatives (with respect to time and space), $I_{t}^{\prime}, I_{x}^{\prime}$ and $I_{y}^{\prime}$ along the propagation path (Davies, 1969; Kolosov et al., 1969; Yakovlev, 1985; Goodman and Aarons, 1990; Yakubov, 1997).

TEC variations may be arbitrarily classified as regular and irregular. Regular changes (seasonal, diurnal) - for the magnetically quiet midlatitude ionosphere at least - are described by models providing relative accuracy of TEC prediction in the range $50 \ldots 80 \%$ (Klobuchar, 1986; Afraimovich et al., 2000).

Irregular changes (variations) are associated with ionospheric irregularities of a different nature, the spectrum of which has a power law character (Gajlit, 1983; Gershman et al., 1984; Yakubov, 1997).
TEC variations introduce proportionate changes of the signal phase $\varphi(t, x, y)=k_{1} I(t$, $x, y)$, which gives rise to measuring errors of the range $\sigma(D)=k_{2} d I$, the frequency Doppler shift of the signal $\sigma(f)=k_{3} I_{t}^{\prime}$, and the angles of arrival of the radio wave $\sigma\left(\alpha_{x}\right)=k_{4} I_{x}^{\prime}$ and $\sigma\left(\alpha_{y}\right)=k_{4} I_{y}^{\prime}$, because the last four quantities are proportional to the time and space derivatives of the phase. Furthermore, the maximum value of the measuring error of angular deviations can be deduced using the relation

$$
\sigma(\alpha)=k_{4} \sqrt{\left(I^{\prime}{ }_{x}\right)^{2}+\left(I^{\prime}{ }_{y}\right)^{2}} .
$$

The coefficients $k_{1}-k_{4}$ are inversely proportional to the signal frequency $f_{\mathrm{c}}$ or to its square (Davies, 1969; Kravtsov et al., 1983; Goodman and Aarons, 1990). A calculation uses a Cartesian topocentric coordinate system with the axis $x$ pointing eastward $\mathrm{E}$, and the axis $y$ pointing northward $\mathrm{N}$.

Investigations of phase fluctuations of transionospheric signals have been and are carried out using radio beacons on satellites with circular and geostationary orbits (Komrakov and Skrebkova, 1980; Livingston et al., 1981; Evans and Wand, 1983; Gajlit, 1983). The trouble with these measurements is that temporal and spatial resolution is low, and continuity and global coverage of observations are unavailable.

Some research results on the prediction and estimation of radio signal fluctuations and errors of RESS performance caused by them were reported in an earlier work (Afraimovich and Karachenschev, 2002).

Results of experimental investigations of phase fluctuations of RESS signals that are discussed in this paper were derived from analyzing the data for a set consisting of 100 to 300 GPS stations covering the time interval 19982002, for different conditions of geomagnetic activity with the $D s t$-index from - 134 to -377 nT (see table I). The number of passes of GPS satellites with a duration of no less than $2.5 \mathrm{~h}$, the data from which were used in the analysis, totaled no less than 700000 , or several orders of magnitude in excess of the currently available statistics of such measurements.

Below we give an outline of the techniques used in this study and illustrate their application 
Table I. General information on the experiment.

\begin{tabular}{|c|c|c|c|c|c|}
\hline N. & Year & $\begin{array}{l}\text { Numbers } \\
\text { of days }\end{array}$ & $\begin{array}{l}\text { Maximum } \\
\text { of } D s t, \mathrm{nT}\end{array}$ & $\begin{array}{l}\text { Numbers of } \\
\text { power spectra }\end{array}$ & $\left\langle\bar{v}_{\sigma(D)}\right\rangle$ \\
\hline \multicolumn{6}{|c|}{ Magnetically disturbed conditions } \\
\hline 1 & 1998 & 11 & -233 & 253 & \multirow{6}{*}{1.95} \\
\hline 2 & 1999 & 5 & -134 & 115 & \\
\hline 3 & 2000 & 11 & -321 & 253 & \\
\hline 4 & 2001 & 13 & -377 & 299 & \\
\hline 5 & 2002 & 9 & -294 & 207 & \\
\hline $\mathrm{N}$ & & 49 & & 1127 & \\
\hline \multicolumn{6}{|c|}{ Magnetically quiet conditions } \\
\hline 1 & 1998 & 13 & 17 & 299 & \multirow{6}{*}{2.10} \\
\hline 2 & 1999 & 7 & 20 & 161 & \\
\hline 3 & 2000 & 10 & 24 & 230 & \\
\hline 4 & 2001 & 11 & 34 & 253 & \\
\hline 5 & 2002 & 12 & 29 & 276 & \\
\hline $\mathrm{N}$ & & 53 & & 1219 & \\
\hline Total & & 102 & & 2346 & \\
\hline
\end{tabular}

in the analysis of ionospheric effects. The overall sample statistics of errors $\sigma(D), \sigma(f)$ and $\sigma(\alpha)$ is presented for different geomagnetic conditions. To ease comparison with other research results reported in Gajlit (1983) and Kravtsov et al. (1983), the errors $\sigma(D), \sigma(f)$ and $\sigma(\alpha)$ are calculated for the working frequency of $300 \mathrm{MHz}$.

\section{General information about the database used}

This study relies on the data from the global network of receiving GPS stations available on the Internet (fig. 1). As is evident from fig. 1, the receiving sites are relatively dense on the territory of North America and Europe, and less as dense in Asia. Fewer stations are located on the Pacific and Atlantic.

Such coverage of the terrestrial surface by GPS receivers already makes it possible to address the problem of a global investigation of ionospheric disturbances and their consequences with a very large spatial accumulation.

Thus, the corresponding number of LOS to the satellite is at least $2000 \ldots 5000$ at any moment of time. This provides a number of statisti- cally independent series at least two orders of magnitude higher than would be realized by recording VHF radio signals from first-generation geostationary satellites or low-orbit navigation satellites, TRANSIT (Evans and Wand, 1983; Gershman et al., 1984).

For a variety of reasons, for the various events to be analyzed, we selected somewhat differing sets of receiving stations, yet the geometry of the experiment was virtually identical for all events. The coordinates of the receiving stations used in the experiment were available at the electronic address ftp://lox.ucsd.edu/pub.processing/gamit/setup/coords.txt.

\section{Analysis of the measuring errors of the range, Doppler frequency and angles of ar- rival of the radio wave caused by changes in the regular ionosphere}

The standard IONEX format is described in detail in (Schaer et al., 1998). Therefore, we will not give a detailed description of the Global Ionospheric Maps technology for reasons of space but limit ourselves only to the information required for the presentation of our method. 


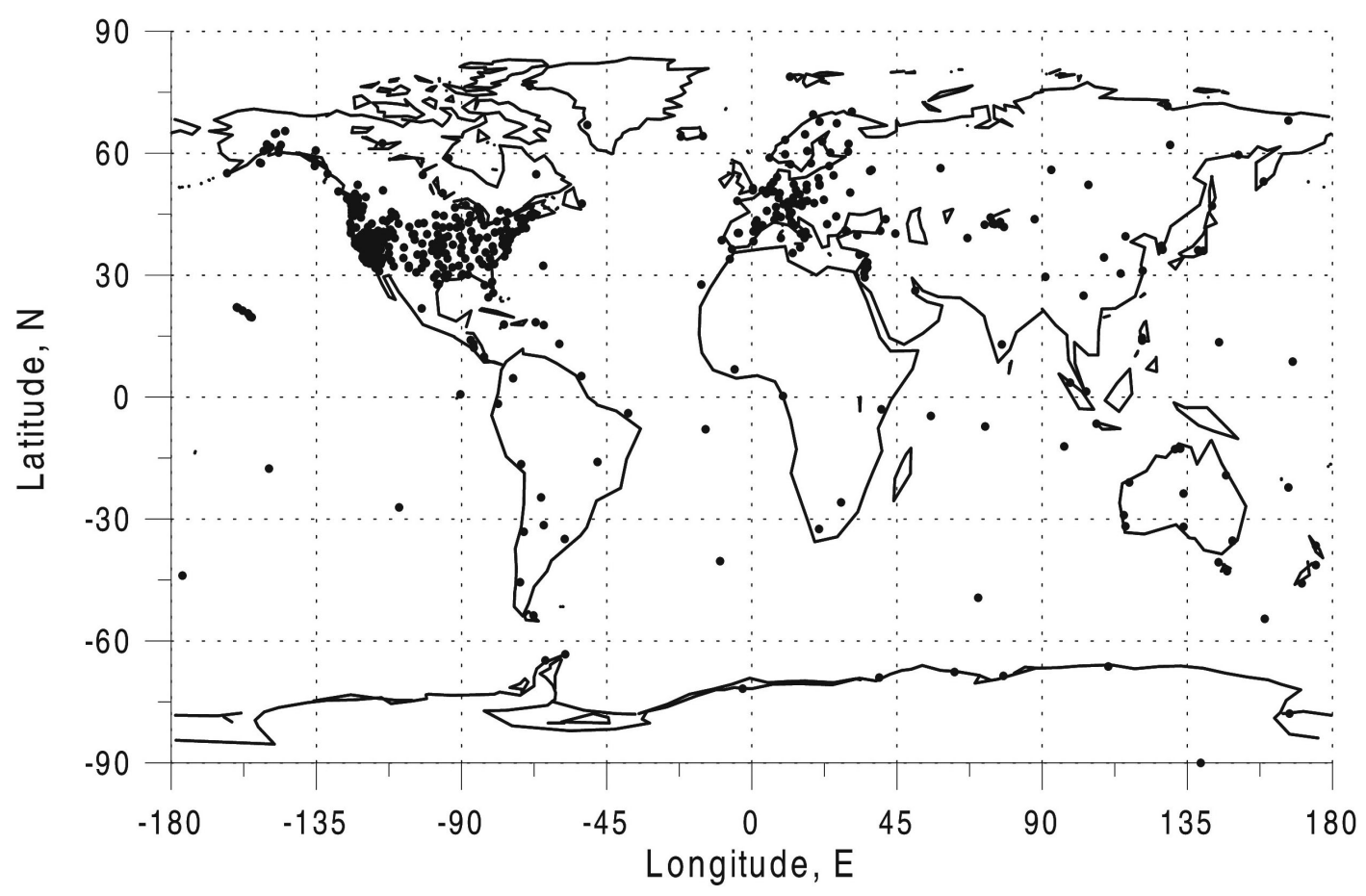

Fig. 1. The map shows the location of the receiving stations of the global GPS network that posts data on the Internet. The number of receiving sites totals more than 1000 as of the beginning of 2002.

Figure 2 is a schematic representation of a single elementary GIM cell. The cell nodes are designated as $a, b, c, d$. The cell size ( $5^{\circ}$ in longitude and $2.5^{\circ}$ in latitude) is determined by the IONEX file standard. For simplifying the transformations to an approximation sufficient for our problem for latitudes not exceeding $60^{\circ}$, the cell can be represented as a rectangle with the sides $d_{e}$ and $d_{n}$. It is easy to overcome this limitation by complicating to a certain extent the transformations allowing for the sphericity; however, we do not present them in this report.

The linear size of the rectangular cell in latitude is independent of the latitude and is $d_{n}=279$ $\mathrm{km}$; the linear size in longitude depends on the latitude, and for $40^{\circ} \mathrm{N}$ it is $d_{e}=436 \mathrm{~km}$.

For each time $t$, for the nodes $a, b, c, d$ from the IONEX file the values of vertical TEC are known, $I_{a}, I_{b}, I_{c}, I_{d}$.
The determination of the range $D$ by the phase method is based on measuring the phase difference $\varphi$ between the received signal and the reference signal formed in the receiver. Such a measurement can be made at the intermediate or carrier frequency of the signal. In this case

$$
D=c \times \frac{\varphi}{2 \pi f_{c}}
$$

where $c$ is the propagation velocity of radio waves in a free space.

Generally the quantity $\varphi$ for the transionospheric propagation may be regarded as the sum of two components (Afraimovich et al., 1998)

$$
\varphi=\varphi_{s}+\Delta \varphi
$$

where $\varphi_{s}$ is the main component associated with a change in the distance between the signal source and the receiver. 


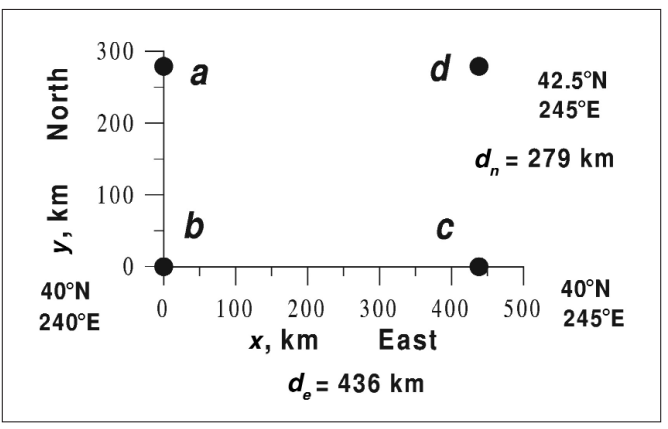

Fig. 2. Geometry of an elementary GIM cell. Nodes of the cell are denoted $a, b, c$ and $d$. The cell size is determined by the values of $d_{n}=279 \mathrm{~km}$ and of $d_{e}$ dependent on the cell's location latitude.

Analysis of the measuring errors of the range, Doppler frequency and angles of arrival of the radio wave caused by changes in the regular ionosphere investigations of global phase variations of radio signals and their influence on the operation of RESS ought to take into account the proportionate relationship between phase (phase derivative) changes of the transionospheric signal and corresponding TEC variations (Kravtsov et al., 1983; Goodman and Aarons, 1990)

$$
\Delta \varphi=8.44 \times 10^{-7} \times \frac{d I_{a}(b, c, d)}{f_{c}}+\varphi_{0}
$$

where $f_{c}$ is the radio wave frequency $(\mathrm{Hz}) ; d I_{a(b}$, $c, d)$ is the TEC variations measured at the points $a, b, c$, and $d\left(10^{16} \mathrm{e} / \mathrm{m}^{2}\right)$; and $\varphi_{0}$ is the initial phase (Spoelstra and Kelder, 1984).

By way of example we now analyze the errors of measurement of the range $\sigma(D)$, the frequency Doppler shift $\sigma(f)$ and the angle of arrival of the radio wave $\sigma(\alpha)$ using the IONEX data and the method that was developed at the ISTP SD RAS (Afraimovich and Karachenschev, 2002).

The change in the range that is introduced by the ionosphere (ionospheric error) is proportional to $\Delta \varphi$

$$
\sigma(D)=c \times \frac{\Delta \varphi}{2 \pi f_{c}}
$$

Upon substituting eq. (4.3) into eq. (4.4), we can obtain the expression for determining the measuring error of the range $\sigma(D)$ introduced by the ionosphere

$$
\sigma(D)=\frac{c \times 8.44 \times 10^{-7} \times d I}{2 \pi f_{c}^{2}}=4.48 \times d I .
$$

As is seen from eq. (4.5), the error $\sigma(D)$ is directly proportional to the TEC variation $d I$ and inversely proportional to the carrier frequency squared.

Using the values of the spatial derivatives of TEC and $I_{x}^{\prime}$ and $I_{y}^{\prime}$ of the derivative of TEC with respect to time $I_{t}^{\prime}$ makes it possible to uniquely obtain - for each instant of time - the values of errors of determination of the angle of arrival $\sigma(\alpha)$ and the frequency Doppler shift $\sigma(f)$ by formulas (Kravtsov et al., 1983; Goodman and Aarons, 1990)

$$
\begin{gathered}
\sigma(\alpha)=\frac{1.39 \times 10^{2}}{f_{c}^{2}} \times \sqrt{\left(I_{x}^{\prime}\right)^{2}+\left(I_{y}^{\prime}\right)^{2}} \\
\sigma(f)=\frac{1.34 \times 10^{-7}}{f_{c}} \times I_{t}^{\prime} .
\end{gathered}
$$

In the simplest case, the values of the derivatives for the selected cell of the map can be obtained using TEC increments for the four cell nodes and for two times $t_{1}$ and $t_{2}=t_{1}+d_{t}$

$$
\begin{aligned}
& d I=\left(I_{a 2}-I_{a 1}+I_{b 2}-I_{b 1}+I_{c 2}-I_{c 1}+I_{d 2}-I_{d 1}\right) / 4 \\
& I_{t}^{\prime}=\left(I_{a 2}-I_{a 1}+I_{b 2}-I_{b 1}+I_{c 2}-I_{c 1}+I_{d 2}-I_{d 1}\right) / 4 d_{t} \\
& I_{x}^{\prime}=\left(I_{c 1}-I_{b 1}+I_{d 1}-I_{a 1}+I_{c 2}-I_{b 2}+I_{d 2}-I_{a 2}\right) / 4 d_{e} \\
& I_{y}^{\prime}=\left(I_{a 1}-I_{b 1}+I_{d 1}-I_{c 1}+I_{a 2}-I_{b 2}+I_{d 2}-I_{c 2}\right) / 4 d_{n} .
\end{aligned}
$$

Where necessary, the spatial derivatives can be estimated by taking into account the TEC values in adjacent nodes of the map, and the time derivative (with a time resolution of IONEX maps no worse than $15 \mathrm{~min}$ ) can be inferred by averaging increments for several successive time counts.

The procedures (4.5), (4.6) and (4.7) are performed for all cells of the selected spatial range 
and for the selected time interval of the day. One variant of data representation implies a full exploitation of the IONEX format with the difference that, rather than the values of TEC and the error of TEC determination (Schaer et al., 1998), are entered into the corresponding cells of the map values of error $\sigma(D), \sigma(f)$ and $\sigma(\alpha)$.

By way of example, it is appropriate to give the results derived from analyzing the regular part of the spatial-temporal TEC variations for a relatively magnetically quiet day of July 29, 1999 (with the largest deviation of the Dst-index of $-40 \mathrm{nT}$ ) and for the magnetically disturbed day of April 6, 2000 (with the largest deviation of the Dst-index of - $293 \mathrm{nT}$ ).

Figure 3a-c portrays the maps of the errors $\sigma$ $(D), \sigma(f)$ and $\sigma(\alpha)$ obtained on the basis of files in the IONEX format for the magnetically quiet day of July 29, 1999 in the geographic coordinate system in the range of longitudes $-120^{\circ} \ldots-60^{\circ} \mathrm{E}$ and latitudes $20^{\circ} \ldots 70^{\circ} \mathrm{N}$. Figure $3 \mathrm{~d}-\mathrm{f}$, respectively, characterizes the values of $\sigma(D), \sigma(f)$ and $\sigma(\alpha)$ for the magnetically disturbed day of April 6,2000 . The figure also shows the time interval 19-21 UT, for which the analysis was carried out. Contours show the values of errors of phase measurements in units, respectively, of $\sigma(D)-$ «m» (meters), $\sigma(f)-\langle\mathrm{Hz} »($ Hertz), and $\sigma(\alpha)-$ «arcmin» (minutes of arc). The vertical calibrated scale shows the maximum and minimum values of the corresponding errors.

The above maps are a pictorial rendition of the behavior dynamics of the errors $\sigma(D), \sigma(f)$ and $\sigma(\alpha)$ in the spatial and temporal ranges selected. Noteworthy is a considerable difference of the maps for the magnetically quiet and disturbed days. As is evident from fig. 3a-f, gradients of spatial distribution of the errors during disturbances increase more than an order of magnitude when compared to the quiet period, which would lead to a degradation of RESS performance.

\section{Analysis of the irregular errors $\sigma(D), \sigma(f)$ and $\sigma(\alpha)$ for the North-American sector}

Our analysis of the irregular component of the errors was based on using raw data in the form of series of TEC values for selected receiving sites as well as values of elevations $\Theta_{s}(t)$ and azimuths $\alpha_{s}(t)$ to visible satellites that were calculated using a specially developed program, CONVTEC, to convert RINEX-files (standard files for the GPS system) available from the Internet (Afraimovich et al., 1998).

Figure 4 presents the geometry of transionospheric radio sounding. The axes $z, y, x$ are pointing, respectively, to the zenith, the north $\mathrm{N}$, and to the east $\mathrm{E} ; P$ is the point of intersection of the LOS to the satellite (the line connecting the satellite to the radio signal receiver) with the ionospheric $F_{2}$ region peak; $S$ is the subionospheric point (projection of the point $P$ in to the terrestrial surface).

The GPS technology provides the means of estimating TEC variations on the basis of phase measurements of TEC $I_{0}$ in each of the spaced two-frequency GPS receivers using the formula (Hofmann-Wellenhof et al., 1992)

$$
I_{0}=\frac{1}{40.308} \frac{f_{1}^{2} f_{2}^{2}}{f_{1}^{2}-f_{2}^{2}}\left[\left(L_{1} \lambda_{1}-L_{2} \lambda_{2}\right)+\text { const }+n L\right]
$$

where $L_{1} \lambda_{1}$ and $L_{2} \lambda_{2}$ are phase path increments of the radio signal, caused by the phase delay in the ionosphere (m); $L_{1}$ and $L_{2}$ are the number of full phase rotations, and $\lambda_{1}$ and $\lambda_{2}$ are the wavelengths $(\mathrm{m})$ for the frequencies $f_{1}$ and $f_{2}$, respectively; const is some unknown initial phase path (m); and $n L$ is the error in determination of the phase path $(\mathrm{m})$.

Series of the values of elevations $\Theta_{s}(t)$ and azimuths $\alpha_{s}(t)$ of the beam to the satellite were used to determine the coordinates of subionospheric points, and to convert the «oblique» TEC $I_{0}$ to the corresponding value of the «vertical» TEC $I$ by employing the technique reported by Klobuchar (1986)

$$
I=I_{0} \times \cos \left[\arcsin \left(\frac{R_{z}}{R_{z}+h_{\max }} \cos \Theta_{s}\right)\right]
$$

where $R_{z}$ is the Earth's radius, and $h_{\max }=300$ $\mathrm{km}$ is the height of the $F_{2}$ layer maximum.

All results in this study were obtained for elevations $\Theta_{s}(t)$ larger than $30^{\circ}$.

To analyze the errors $\sigma(D), \sigma(f)$ and $\sigma(\alpha)$ that are caused by the irregular component of 
July 29, 1999

April 6, 2000

Period of times: 19 - 21 UT
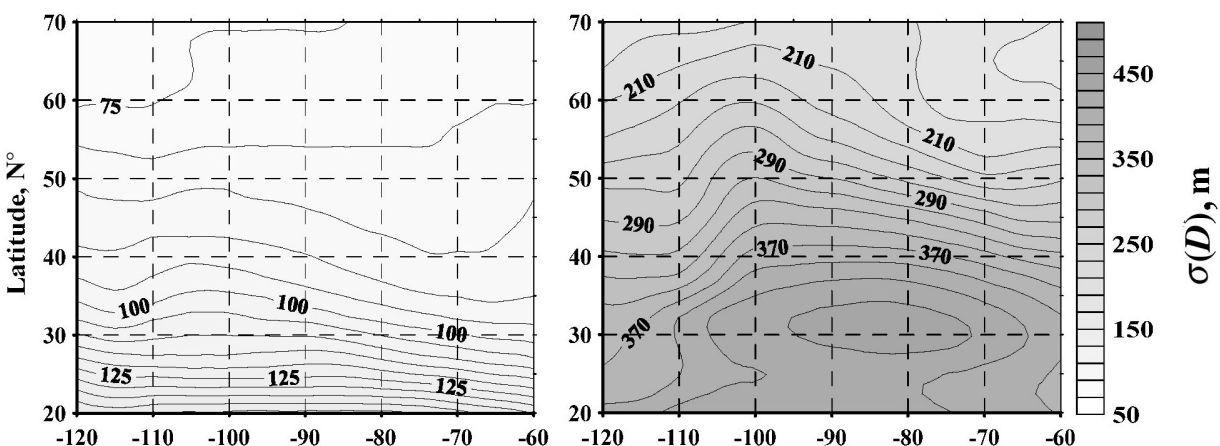

(a)

(d)
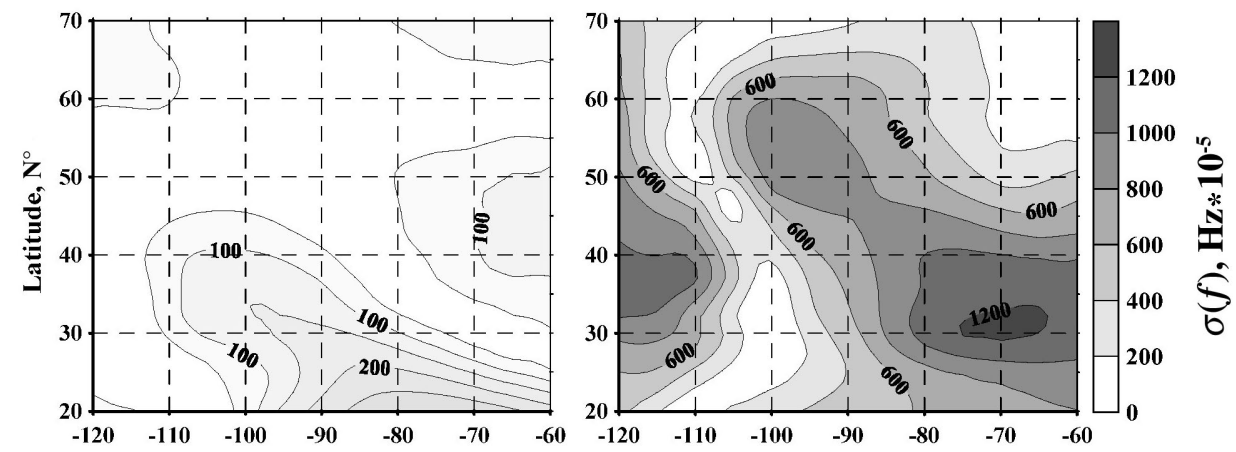

(b)

(e)
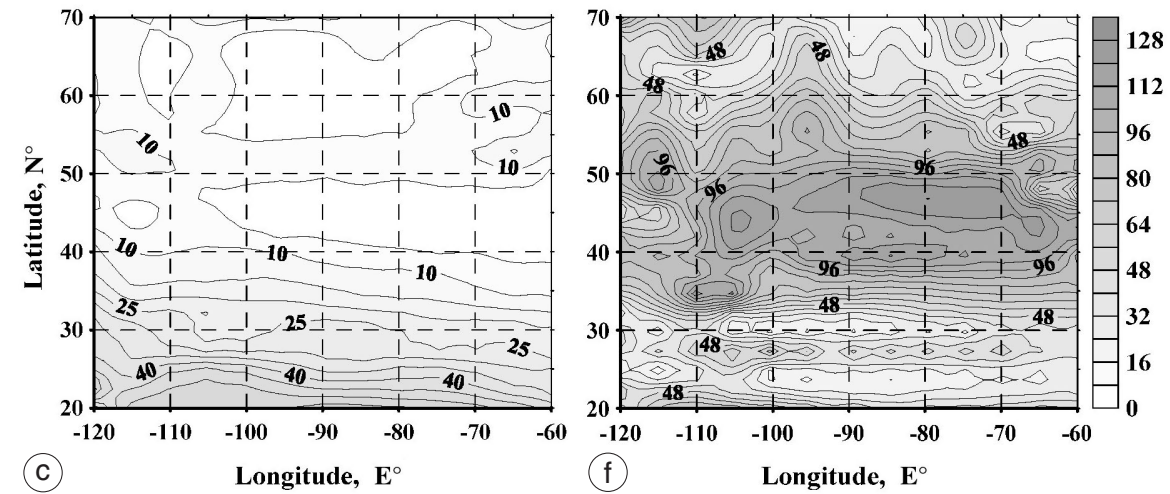

ชै

Fig. 3a-f. Maps of global distribution of measurement errors of the range $\sigma(D)$, frequency Doppler shift $\sigma(f)$, and of angles of arrival of radio waves $\sigma(\alpha)$ (panels a-c, for a magnetically quiet day; panels d-f, for a magnetically disturbed day). The errors can be evaluated from the graduated scale, shown in the figure at the right. 


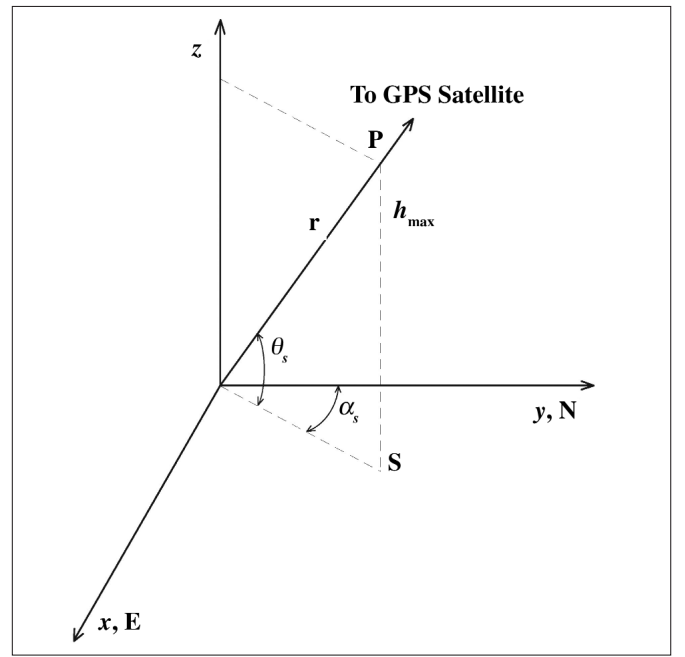

Fig. 4. Schematic representation of the transionospheric sounding geometry. The axes $z, y$ and $x$ are directed, respectively, zenithward, northward $(\mathrm{N})$ and eastward (E); $P$ - point of intersection of Line-OfSight (LOS) to the satellite with a horizontal plane at the height of the maximum of the ionospheric $F_{2}$ region $\left(h_{\max }\right) ; S$ - subionospheric point; $\Theta_{\mathrm{s}}$ and $\alpha_{\mathrm{s}}-$ elevation and azimuth of the direction $\mathbf{r}$ along LOS to the satellite.

TEC variation, we make use of the relations for the respective regular errors (4.5), (4.6) and (4.7). The difference in the analysis of irregular errors implies a different (compared with the IONEX technique) method of determining the values of TEC and its derivatives (Afraimovich and Karachenschev, 2002).

The phase differences $\Delta \varphi_{x, y}$ along the axes $x$ and $y$ are proportional to the values of the horizontal components of TEC gradient $G_{E}=I_{x}^{\prime}$ and $G_{N}=I_{y}^{\prime}$

To calculate the components of the TEC gradient $I_{x}^{\prime}$ and $I_{y}^{\prime}$ are used linear transformations of the differences of the values of the filtered TEC $\left(I_{B}-I_{A}\right)$ and $\left(I_{B}-I_{C}\right)$ at the receiving points $A, B$ and $C$

$$
\begin{aligned}
& I_{x}^{\prime}=\frac{y_{A}\left(I_{B}-I_{C}\right)-y_{C}\left(I_{B}-I_{A}\right)}{x_{A} y_{C}-x_{C} y_{A}} \\
& I_{y}^{\prime}=\frac{x_{C}\left(I_{B}-I_{A}\right)-x_{A}\left(I_{B}-I_{C}\right)}{x_{A} y_{C}-x_{C} y_{A}}
\end{aligned}
$$

where $x_{A}, y_{A}, x_{C}, y_{C}$ are the coordinates of the sites $A$ and $C$ in the topocentric coordinate system (Afraimovich et al., 1998). When deriving (5.3) we took into account that $x_{B}=y_{B}=0$, since site $B$ is the center of topocentric coordinate system.

The time derivative of TEC $I_{t}^{\prime}$ is determined by differentiating $I$ at the point $B$.

The procedures of (4.5), (4.6) and (4.7) are performed for all groups of three GPS stations of the selected spatial range and for satellites visible from these stations, as well as for the selected time interval of the day.

Figure 5a-h presents the results derived from analyzing the irregular component of the errors $\sigma(D), \sigma(f)$ and $\sigma(\alpha)$ in the form of fluctuation spectra of the range, Doppler frequency and angles of arrival of radio waves.

With the purpose of improving the statistical reliability of the data, we used the spatial averaging technique for spectra within the framework of a novel technology (Afraimovich et al., 2001). The method implies using an appropriate processing of TEC variations that are determined from the GPS data, simultaneously for the entire set of GPS satellites (as many as 5-10 satellites) «visible» during a given time interval, at all stations of the global GPS network used in the analysis.

Individual spectra of the errors $\sigma(D), \sigma(f)$ and $\sigma(\alpha)$ were obtained by processing continuous series of $I(t)$ measurements of a duration no shorter than $2.5 \mathrm{~h}$. To eliminate errors caused by the regular ionosphere, as well as trends introduced the motion of satellites, we used the procedure of removing the linear trend by preliminarily smoothing the initial series with the selected time window of a duration of about $120 \mathrm{~min}$.

Figure 5a-h shows the overall character of the TEC variations $d I(t)$ that were filtered from TEC series obtained from measurements of the phase difference between two coherently coupled signals from the GPS system (HofmannWellenhof, 1992) for the magnetically quiet day of July 29, 1999 (panel a, at the left) and a major magnetic storm of July 15, 2000 (panel e, at the right). Furthermore, the panels show the station names and locations, as well as GPS satellite numbers (PRN).

As is evident from the figure, the intensity $d I(t)$ during the disturbance even at such low 


\section{North-American sector $\left(30^{\circ}-60^{\circ} \mathrm{N}, 240^{\circ}-300^{\circ} \mathrm{E}\right)$ \\ July 29, 1999 \\ July 15, 2000}

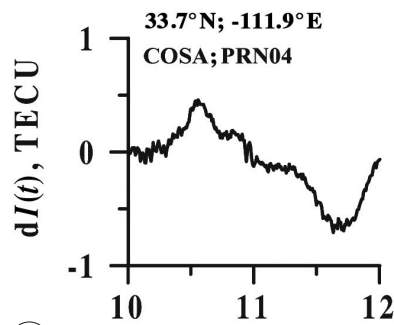

(a)

Time, UT

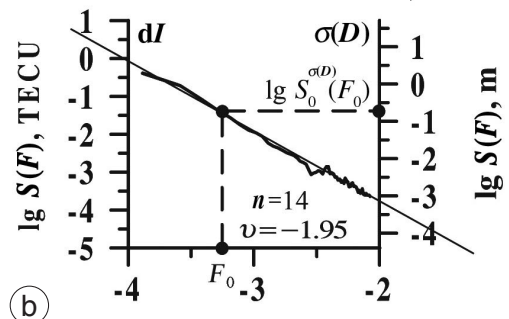

(b)
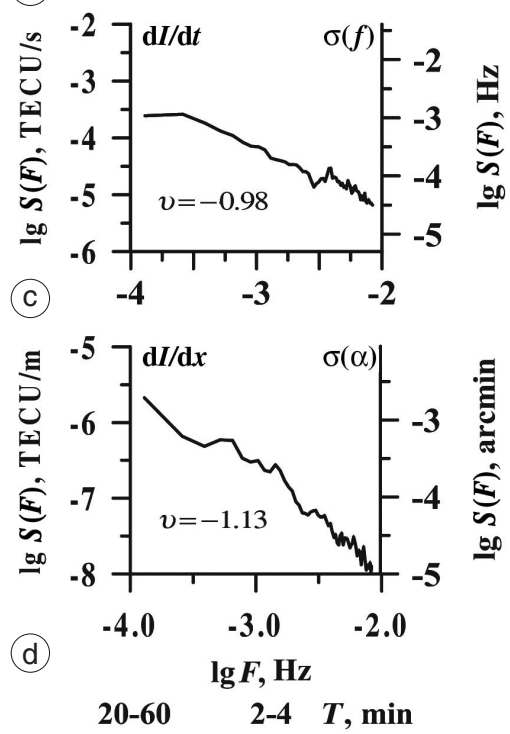

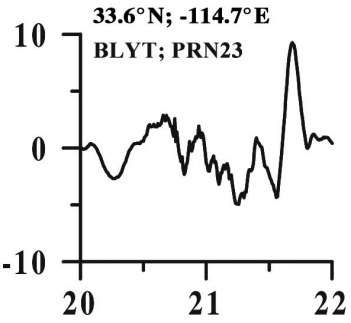

Time, UT

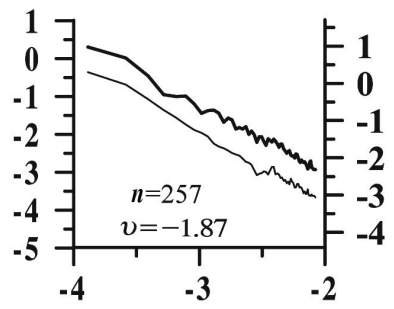

(g)
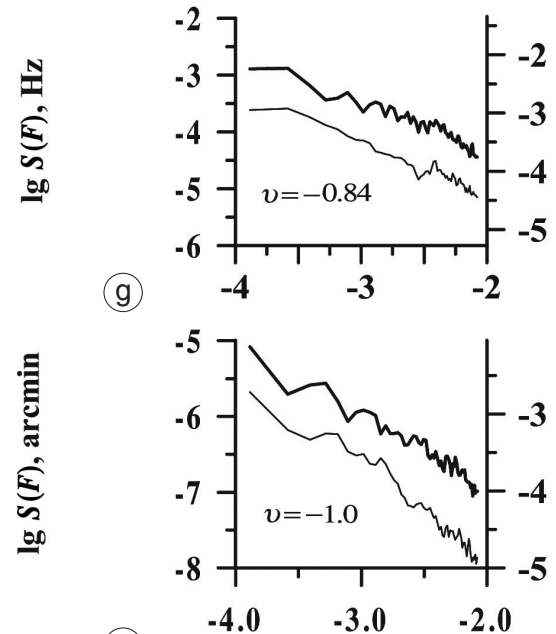

(h)

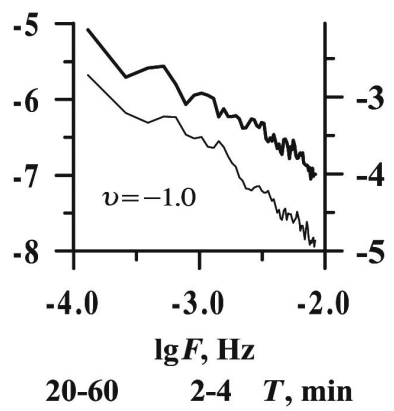

Fig. 5a-h. TEC variations $d I(t)$ for the magnetically quiet day of July 29, 1999 (left, panel a) and a major magnetic storm of July 15, 2000 (right, panel e) for North-American sector. These panels also show the names and coordinates of the COSA and BLYT stations and GPS satellite numbers (PRN). Panels b- $\mathrm{d}$ and f-h on a logarithmic scale present the amplitude spectra $S(F)$ of TEC fluctuations and derivatives (left scale in the panels), and the fluctuation spectra of $\sigma(D), \sigma(f)$ and $\sigma(\alpha)$ converted to the working frequency of $300 \mathrm{MHz}$ (right panel). For comparison, spectra from the magnetically quiet day of July 29, 1999 are shown in panels f-h by the thin line. The range of fluctuation periods is shown in bold type along the abscissa axis of panels d,h. Panels b,e show the number $n$ of GPS arrays. The power law character of the spectra is determined by the values of slopes $v$. The thin black slant line in panel $\mathrm{b}$ is a fitted straight line of the power law spectrum. 
latitudes is increased an order of magnitude as a minimum. This is reflected on logarithmic amplitude spectra $\lg S(F)$ of TEC fluctuations and their derivatives (left-hand scale in the figure) and of the fluctuations of $\sigma(D), \sigma(f)$ and $\sigma(\alpha)$, converted to the working frequency of $300 \mathrm{MHz}$ (right-hand scale) which are represented on a logarithmic scale (panels b-d and panels f-h).

The logarithmic amplitude spectrum $\lg S(F)$ obtained by using a standard FFT procedure. Incoherent summation of the partial amplitude spectra $S_{i}(F)$ of different LOS was performed by the formula

$$
\lg \langle S(F)\rangle=\lg \left[\frac{\sum_{i=1}^{n} S_{i}(F)}{n}\right]
$$

where $i$ is the number of LOS; $i=1,2, \ldots n$.

As a consequence of the statistical independence of partial spectra, the signal/noise ratio, when the average spectrum is calculated, increases due to incoherent accumulation at least by a factor of $\sqrt{n}$, where $n$ is the number of LOS.

Fluctuation spectra from the magnetically quiet day of July 29, 1999 are shown by the thin line in panels f-h (fig. 5) for comparison with the spectra from the disturbed day. The range of fluctuation periods is shown in bold type along the abscissa axis in panels $d$ and $h$. Panels $b$ and f show also the number $n$ of GPS arrays composed of three stations, the data from which are used to estimate the spatial derivatives of TEC (Afraimovich et al., 2001).

Spectra of phase fluctuations have a power law character with the values of the slopes $v$, shown in panels b-d and $\mathrm{f}-\mathrm{h}$. The slope of spectrum is determined by the slope of the fitted straight line (thin black line in panel b of fig. 5). These results are in reasonably good agreement with data reported in (Komrakov and Skrebkova, 1980; Livingston et al., 1981; Gajlit et al., 1983; Kravtsov et al., 1983; Gershman et al., 1984; Yakubov, 1997).

The superposition of Travelling Ionospheric Disturbances (TIDs) of different origins that are responsible for the range of periods of 10-120 min in the spectrum of TEC disturbances. TIDs are of great interest as a matter for scientific inquiry in the ionosphere physics and as a factor that limits the accuracy of modern-day radio engineering systems used in navigation and radio interferometry; a rich variety of publications addressed the issue of TIDs (Evans and Wand, 1983; Hocke and Schlegel, 1996; Oliver et al., 1997; Afraimovich et al., 1998; etc).

TIDs are classified as Large-Scale and Medium-Scale disturbances (LSTIDs and MSTIDs). LSTIDs have horizontal phase speeds between 400 and $1000 \mathrm{~m} / \mathrm{s}$ (comparable with the sound velocity in the thermosphere), horizontal wavelengths greater than $1000 \mathrm{~km}$ and periods in the range of $30 \mathrm{~min}$ to $3 \mathrm{~h}$. LSTIDs are predominant in the night-time hours and are closely associated with geomagnetic and auroral activity.

MSTIDs have horizontal phase velocities between 100 and $250 \mathrm{~m} / \mathrm{s}$ (less than the sound velocity in the lower atmosphere), wavelengths of several hundred kilometers and periods between 15 and $60 \mathrm{~min}$. MSTIDs are observed predominantly during the daytime hours and are associated with AGW which are possibly generated in the lower atmosphere.

It is known that the sources of MSTIDs can include natural processes of a different origin: magnetic storms, auroral phenomena, dynamic processes in the lower and middle atmosphere (e.g., convection, orography, shear flow, jet stream), the solar terminator, strong earthquakes, volcanic eruptions, as well as anthropogenic influences (rocket launchings, explosions, nuclear tests). As a consequence the observed picture of the electron density disturbances is essentially a net interference wave field of AGWs of different origins and their interactions with the ionospheric plasma. Identifying of the AGW of a definite type from this field is a highly involved and generally an almost intractable problem.

\section{Analysis of the irregular errors $\sigma(D), \sigma(f)$ and $\sigma(\alpha)$ for the Western Europe sector}

As stated above, this paper presents the results obtained for the North-American sector of the terrestrial surface, which is due to the avail- 
ability of a denser network of stations in this region. On the other hand, the proposed technique can be used to estimate errors in any other region. However, the statistical reliability of the results of estimation will depend directly on the number and relative location of receiving sites in the region of our interest.

As an example, at this point we present the results from estimating the errors obtained for the West-European region $\left(30-60^{\circ} \mathrm{N}, 0-45^{\circ} \mathrm{E}\right)$. Figure 6a-h shows, as power spectra, the results from estimating the errors for two days: magnetically quiet July 15,2001 , and magnetically disturbed July 15,2000 . As is seen from the figure, the variation of $d I(t)$ during the disturbance (fig. 6e) exceeds more than by a factor of two that for a quiet day (fig. 6a).

The somewhat smaller values of $d I(t)$ when compared with the results presented in fig. 5a$\mathrm{h}$, may be explained as follows. The Earth's geomagnetic pole is displaced with respect to the geographic pole. As a consequence of this, the ionospheric properties for the North-American region are somewhat altered when compared with the region of West Europe.

At the latitudes under analysis, the ionosphere in West Europe has the properties of the mid-latitude ionosphere, whereas in the NorthAmerican sector at the same geographic latitudes the ionosphere approaches, in its properties, the high-latitude ionosphere. This fact explains certain differences of the value of $d I(t)$ for the two regions under consideration.

However, even in this case the values of irregular TEC fluctuations and their derivatives (left-hand scale in the figures) and of the fluctuations of $\sigma(D), \sigma(f)$ and $\sigma(\alpha)$, converted to the working frequency of $300 \mathrm{MHz}$ (right-hand scale) during the disturbance (panels f-h) exceed those for quiet conditions (panels b-d) by a factor of 5-7, as a minimum.

The necessary stage of investigation involves obtaining the latitude-dependence of the values of the errors $\sigma(D), \sigma(f)$ and $\sigma(\alpha)$. Figure $7 \mathrm{a}-\mathrm{h}$ presents the results from estimating the errors for two latitude ranges in Western Europe during a major magnetic storm on July 15 , 2000: the low-latitude region $\left(30-45^{\circ} \mathrm{N}\right.$, 0$\left.50^{\circ} \mathrm{E}\right)$ and high-latitude region $\left(65-75^{\circ} \mathrm{N}, 0-\right.$ $\left.50^{\circ} \mathrm{E}\right)$. It is evident from the figure that the high-latitude variation of $d I(t)$ (fig. 7e) exceeds by more than a factor of four that for the lowlatitude region (fig. 7a).

However, such a variation has had a little effect on the intensity of the power spectrum, especially in the region of low-frequency fluctuations of the errors. On the other hand, the amplitude of the high-frequency components of the errors changed more drastically, which led to some changes in the slopes of the power spectra (fig. 7f-h).

\section{Distribution of slopes and scales of spectra of the errors}

In the preceding section we obtained the fluctuation spectra of the errors $\sigma(D), \sigma(f)$ and $\sigma(\alpha)$ corresponding to different ionospheric conditions. It was shown that the spectra have a power law character, with definite indices of the slope $v$ (fig. 5a-h). However, the individual spectra obtained do not give a full insight into the global picture of fluctuations of the errors. To obtain a generalized estimate, we carried out an analysis of the spectra of errors obtained by processing the data covering 102 days (over 2346 spectra).

In our experiment, the range of fluctuation periods of spectra varies from 2 min to $2 \mathrm{~h}$. Experimental spectra of the errors $\sigma(D), \sigma(f)$ and $\sigma(\alpha)$ have a complicated form (figs. 5a-h and $6 \mathrm{a}-\mathrm{h})$. There are spectral density maxima and minima. Generally, however, the spectral density $S(F)$ decreases with the increasing fluctuation frequency. For each individual spectrum, calculated on a logarithmic scale, we determined the fitted straight line.

The result of a generalized estimation is represented by the plots in fig. 8a-c. The plots show the distributions of the slopes $P_{\sigma(D)}(v)$, $P_{\sigma(f)}(v), P_{\sigma(\alpha)}(v)$, of power law spectra of the errors $\sigma(D), \sigma(f)$ and $\sigma(\alpha)$, respectively. The interval $\Delta v$ of the slopes of the spectra $\sigma(D)$ is $\Delta v_{\sigma(D)}=-1.45 \ldots-2.45$, with the mean value of $\left\langle\Delta v_{\sigma(D)}\right\rangle=-1.95$. In view of the relationship between the spectrum $\sigma(D) \sim \varphi(t)$ and the spec$\operatorname{tra} \sigma(f)$ and $\sigma(\alpha)$ that are proportional to the derivative of $\varphi(t, x, y)\left(\sigma(f) \sim \varphi^{\prime}(t)\right.$ and $\sigma(\alpha) \sim$ $\left.\sim \varphi^{\prime}(x, y)\right)$, for the slopes of the spectra $\sigma(f)$ 
Western Europe sector $\left(40^{\circ}-70^{\circ} \mathrm{N}, 0^{\circ}-45^{\circ} \mathrm{E}\right)$

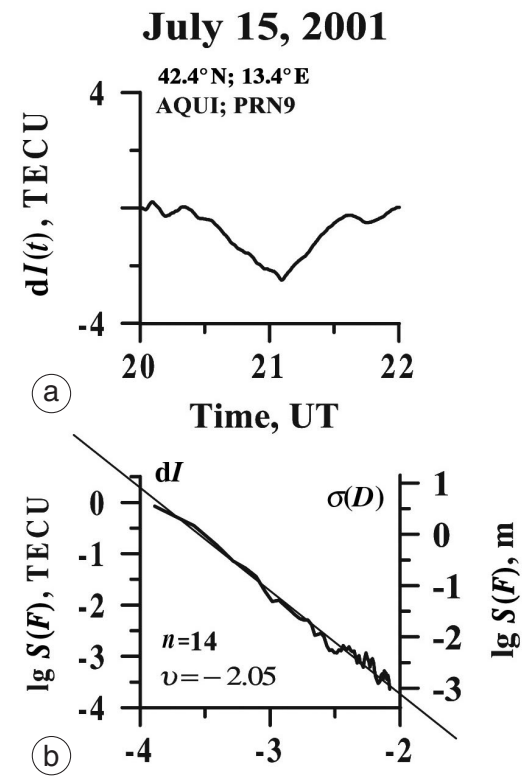

\section{July 15, 2000}

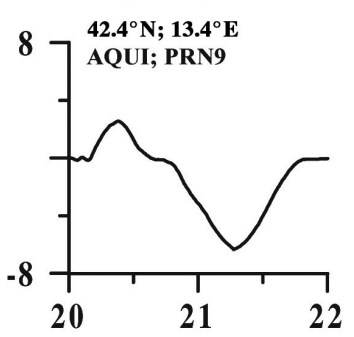

(e)

Time, UT

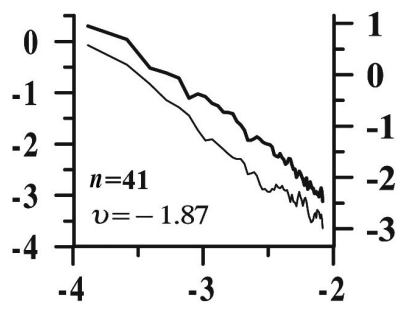

(9)

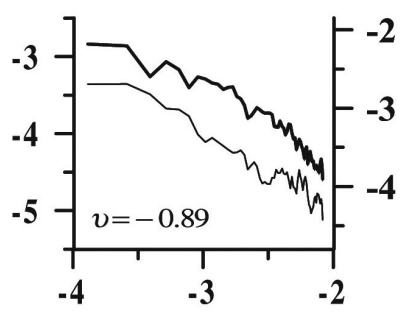

(h)

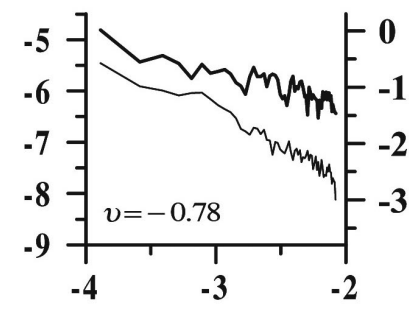

$\lg \mathrm{F}, \mathrm{Hz}$

20-60 2-4 $T$, $\min$

Fig. 6a-h. The dependence of errors of $\sigma(D), \sigma(f)$ and $\sigma(\alpha)$ for the Western Europe sector. TEC variations $d I(t)$, logarithmic scale amplitude spectra $S(F)$ of TEC fluctuations and derivatives (left scale in the panels b-d and f-h), and the fluctuation spectra of $\sigma(D), \sigma(f)$ and $\sigma(\alpha)$ converted to the working frequency of $300 \mathrm{MHz}$ (right scale in the panels b-d and f-h) for the magnetically quiet day of July 15, 2001 (on the left) and a major magnetic storm of July 15, 2000 (on the right). 
Low latitude

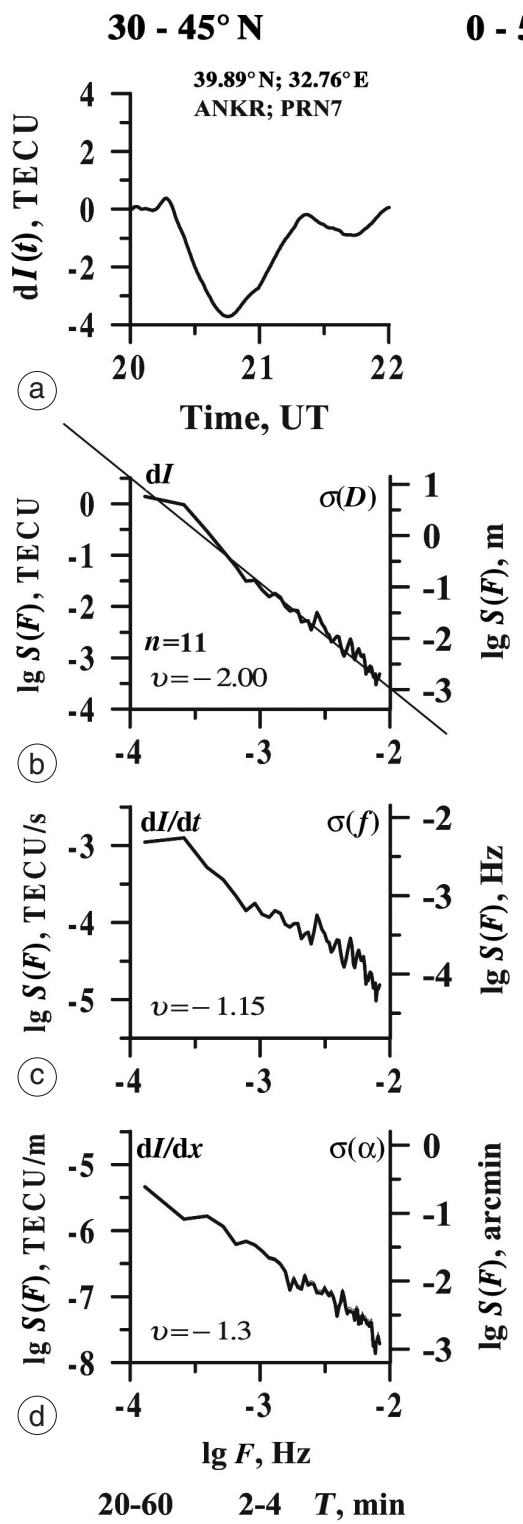

15 July $2000 \quad$ High latitude

$65-75^{\circ} \mathrm{N}$

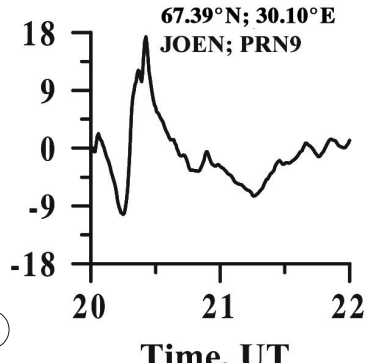

(f)

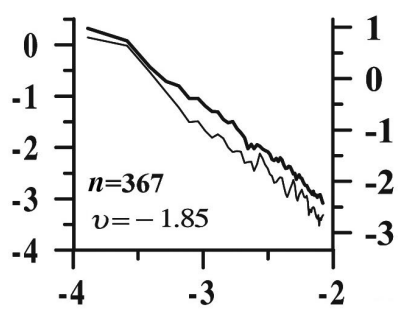

(9)

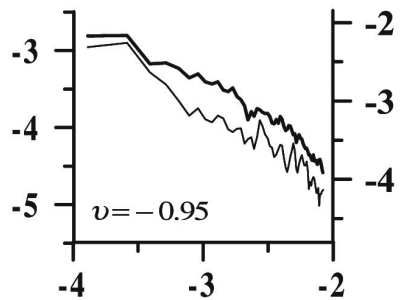

(h)

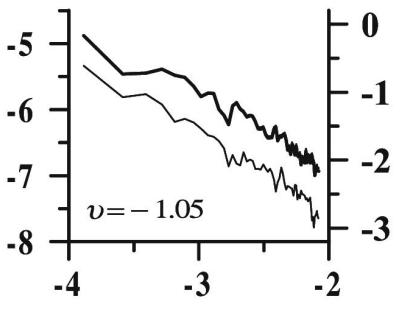

$\lg F, \mathbf{H z}$

20-60 2-4 $T$, min

Fig. 7a-h. TEC variations $d I(t)$, logarithmic scale amplitude spectra $S(F)$ of TEC fluctuations and derivatives (left scale in the panels b-d) and f-h)), and the fluctuation spectra of $\sigma(D), \sigma(f)$ and $\sigma(\alpha)$ converted to the working frequency of $300 \mathrm{MHz}$ (right scale in the panels b-d and f-h) for the lower latitudes (30-45 ${ }^{\circ}$, on the left panels) and a higher latitudes (60-75 , on the right panels) in Western Europe during major magnetic storm July 15, 2000. 
and $\sigma(\alpha)$ these values are, respectively, $\Delta v_{\sigma(f)}=$ $=-0.40 \ldots-1.60,\left\langle\Delta v_{\sigma(f)}\right\rangle=-0.99$ and $\Delta v_{\sigma(\alpha)}=$ $=-0.40 \ldots-2.00,\left\langle\Delta v_{\sigma(\alpha)}\right\rangle=-1.8$.

The aforementioned results derived from estimating the mean values of the slopes of power spectra of the errors neglected the limitations on ionospheric conditions. On the other hand, examination of the individual energy spectra of the errors (figs. 5a-h and $6 a-h$ ) reveals some difference in the slope of the spectra for different levels of ionospheric distur-

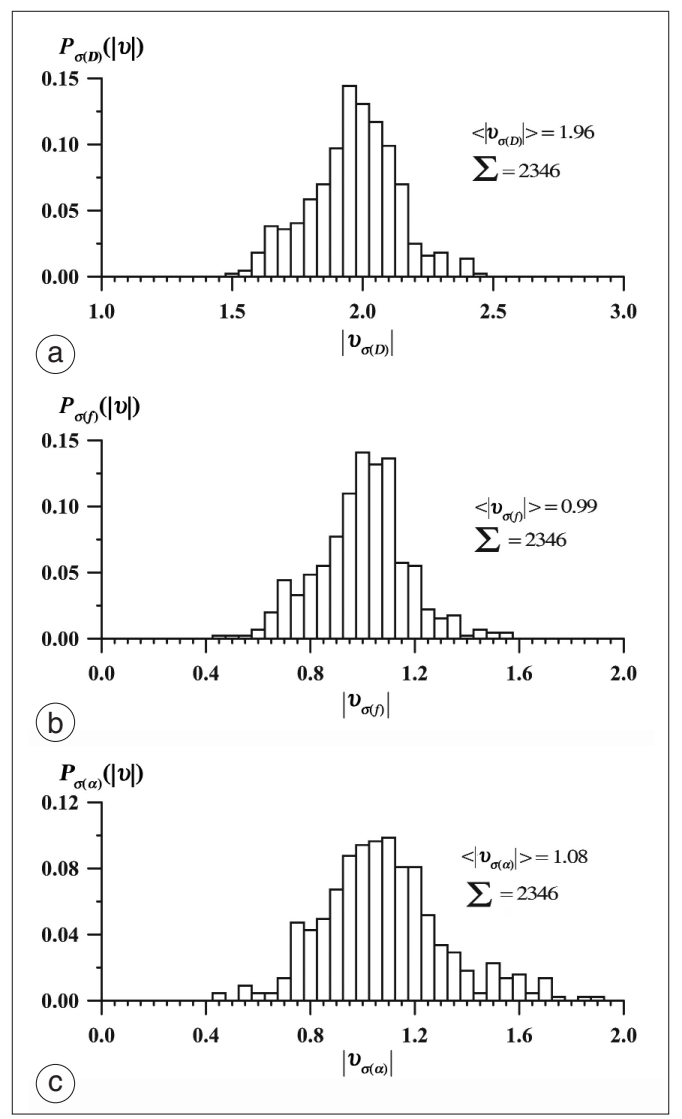

Fig. 8a-c. Probability distributions (a) $P_{\sigma(D)}(v),($ b) $P_{\sigma(f)}(v)$ and (c) $P_{\sigma(\alpha)}(v)$ of the slopes $v_{\sigma(D)}, v_{\sigma(f)}$ and $v_{\sigma(\alpha)}$ of the power law spectra of the errors $\sigma(D), \sigma(f)$ and $\sigma(\alpha)$. Mean values of the slopes $v$, and also total numbers of the values of the slopes of the corresponding spectra use in the analysis are shown in all panels. bance. To investigate this evidence, we analyzed the dependence of the slope of the power spectrum on ionospheric conditions; the statistical sample is given in table I.

The results obtained in experiment are presented in fig. 9a-d in the form of a distribution of the slopes of power spectra of the errors $\sigma(D)$ for different levels of geomagnetic activity. As is apparent from fig. 9a-d, the value of the index of the power spectrum $\sigma(D)$ during the disturbance (fig. 9b) is somewhat smaller when compared with quiet conditions (fig. 9a): see table I.

Besides, the slope index of the power spectrum $v_{\sigma(D)}$ depends on the latitude of the point of observation. Figure 9c,d presents the distributions of the mean value of the slopes of the power spectrum of the error $\sigma(D)$ for two latitude ranges (magnetically disturbed conditions). As is evident, the mean value of the slope of the spectrum $\sigma(D)$ at low latitudes (fig. 9c) is somewhat smaller when compared with that obtained for high latitudes (fig. 9d).

Our results may be explained as follows. Firstly, the change of the mean slope of the power spectrum during the disturbance is associated with the high intensity of the high-frequency component of the spectrum in that period. As a result, the mean slope of the spectrum decreases (fig. $9 \mathrm{a}, \mathrm{b})$. Secondly, irrespective of the level of disturbance, the intensity of small-scale electron density irregularities in high latitudes is higher than that in low latitudes, which also leads to a decrease of the mean slope of the spectrum (fig. 9c,d).

Our results are in good agreement with findings reported by a number of authors (Gdalevich et al., 1980; Gajlit, 1983; Kaliev et al., 1988).

The fitted straight line (the slant thin black line in panel b, fig. 5) for the logarithmic scale of errors in range may be described by the expression (7.1)

$$
Y=a \times X+b
$$

here $a$ characterizes the slope of the straight line, and $b$ is the scale coefficient characterizing the rise of the straight line with respect to the abscissa axis, that is, the value of $Y_{0}$ when $X_{0}=0$.

The value of $\lg S^{\sigma(D)}(F)$ can be determined from a single (previously determined) set of 


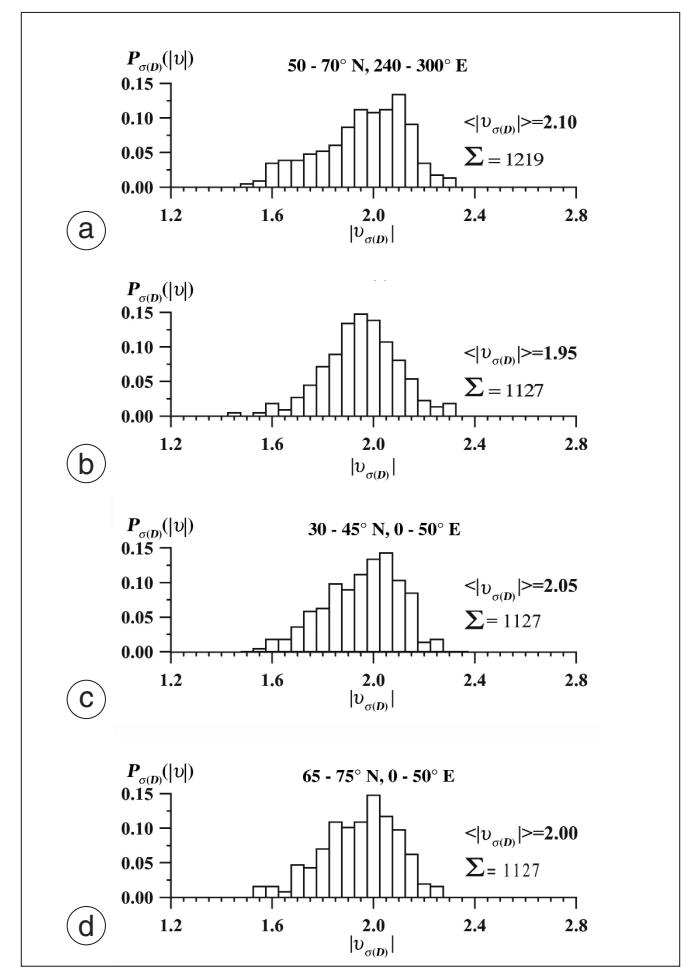

(9)

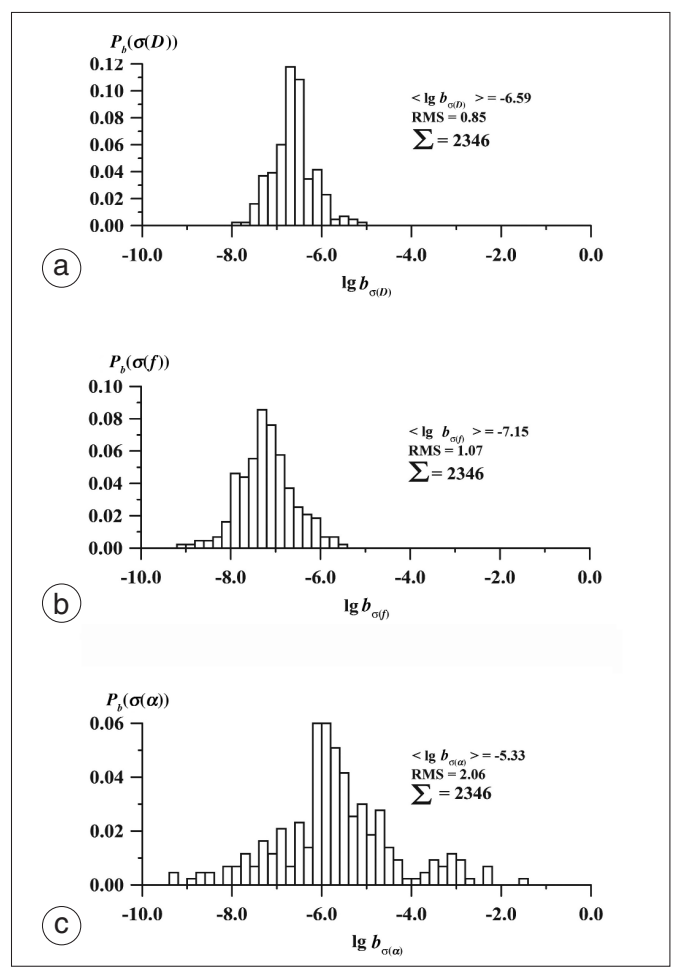

(10)

Fig. 9a-d. Probability distributions means values of the slopes of power law spectra of the error $\sigma(D)$. The $v_{\sigma(D)}$ dependence for different levels of geomagnetic activity: panel (a) (magnetically quiet conditions) and panel (b) (magnetically disturbed conditions). The $v_{\sigma(D)}$ dependence on latitude (magnetically disturbed conditions): panels (c) and (d). Mean values of the slopes $v_{\sigma(D)}$, and also total numbers of the values of the slopes of the corresponding spectra use in the analysis are shown in all panels.

Fig. 10a-c. Probability distributions (a) $P_{b}(\sigma(D))$, (b) $P_{b}(\sigma(f))$ and (c) $P_{b}(\sigma(\alpha))$ of the scales $\lg b_{\sigma(D)}, \lg b_{\sigma(f)}$ and $\lg b_{\sigma(\alpha)}$ of the power law spectra of the errors. Mean values of the slopes, $\left\langle\lg b_{\sigma(D)}\right\rangle,\left\langle\lg b_{\sigma(f)}\right\rangle,\left\langle\lg b_{\sigma(\alpha)}\right\rangle$ (on a $\operatorname{logarithmic}$ scale), standard deviations, and also total numbers of the values of the scales of the corresponding spectra used in the analysis are shown in all panels.

values of $\lg (F), v_{\sigma(D)}$ and $b_{\sigma(D)}$ on the basis of the expression

$$
\lg S^{\sigma(D)}(F)=\nu_{\sigma(D)} \times \lg (F)+b_{\sigma(D)}
$$

Using (7.2) and the resulting value of $b_{\sigma(D)}$ and $v_{\sigma(D)}$ we can determine the value of any spectral component of $\lg S^{\sigma(D)}(F)$ and determine its contribution to the total error. Proceeding in a similar way, we can describe the spectral characteristics of $\sigma(f)$ and $\sigma(\alpha)$.
Panels a-c in fig. 10 present the distributions of the values of the scales of power law spectra of the errors $b_{\sigma(D)}, b_{\sigma(f)}$ and $b_{\sigma(\alpha)}$ obtained for the entire set of data (2346 spectra).

Let us estimate the amplitude $\lg S_{0}^{\sigma(D)}$ of the 32 min harmonic $F_{0}$ of the spectrum of the errors $\sigma(D)$ (fig. 5b) by making use of the values of $\left\langle v_{\sigma(D)}\right\rangle=-1.96$ (fig. 8a) and $\left\langle b_{\sigma(D)}\right\rangle=-6.59$ (fig. 10a). Upon substituting the corresponding values into (7.2), we obtain $\lg S_{0}^{\sigma(D)}\left(F_{0}\right)=-0.153$, which corresponds to $\sigma(D)=0.70[\mathrm{~m}]$. 


\section{Conclusions}

The main results of this study are as follows:

i) This paper suggests a new technique for estimating the errors of RESS performance, based on using phase measurements from two-frequency receivers of the satellite navigation GPS system.

ii) Fluctuation spectra of the errors are generally quite well approximated by the power function. Mean values of the slopes of spectra, obtained from the experiment, are in good agreement with data reported by other authors.

iii) The errors $\sigma(D), \sigma(f)$ and $\sigma(\alpha)$ are convenient estimates with a sufficient accuracy for practical purposes by using mean statistical data for indices of slopes of spectra $v$ and scale coefficients $b$.

iv) Our results can be equally assigned to the estimation of the Russian GNSS-GLONASS and European GNSS-Galileo performance quality.

\section{Acknowledgements}

The authors are indebted to E.A. Kosogorov and O.S. Lesuta for preparing the input data. Thanks are also due V.G. Mikhal- kovsky for his assistance in preparing the English version of the $\mathrm{TE}_{\mathrm{E}} \mathrm{X}$ manuscript. Finally, the authors wish to thank the referees for valuable suggestions which greatly improved the presentation of this paper. This work was done under state support grant No. Nsh-272.2003.5 for Leading Scientific Schools of the Russian Federation as well as Russian Foundation for Basic Research grants No. 00-05-72026 and 0305-64100. We acknowledge the Scripps Orbit and Permanent Array Center (SOPAC) for providing GPS data used in this study.

\section{REFERENCES}

Afraimovich, E.L. (2000): The GPS global detection of the ionospheric response to solar flares, Radio Sci., 35 (6), 1417-1424

AFRAIMOVICH, E.L. and V.A. KARACHENSCHEV (2002): Range, Doppler frequency and radio wave angle-of-arrival measurement errors for satellite-based radio engineering sys- tems caused by the Earth's ionosphere (as deduced using data from the global GPS network), in Proceedings of VIII International Symposium on Radar, Navigation and Communication, Voronezh, vol. 2, 1405-1416.

Afraimovich, E.L., K.S. PALAmartchouK and N.P. PereValOVA (1998): GPS radio interferometry of travelling ionospheric disturbances, J. Atmos. Solar-Terr. Phys., 60 (12), 1205-1223.

Afraimovich, E.L., V.V. Chernukhov and V.V. Demyanov (2000): Updating the ionospheric delay model for singlefrequency equipment of users of the GPS, Radio Sci., 35 (1), 257-262.

Afraimovich, E.L., E.A. Kosogorov, O.S. Lesyuta, A.F. YAKOVETS and I.I. USHAKOV (2001): Geomagnetic control of the spectrum of traveling ionospheric disturbances based on data from a global GPS network, Ann. Geophysicae, 19 (7), 723-731.

DAvIES, K. (1969): Ionospheric Radio Waves (Blaisdell Pub. Co., Waltha, Massachusetts-Toronto-London), pp. 460.

EvANS, J.V. and R.H. WAND (1983): Travelling ionospheric disturbances detected by UHF angle-of-arrival measurements, J. Atmos. Terr. Phys., 65 (2), 122-128.

GaJlit, T.A., V.D. GuSEv, L.M. ERUKHIMOV and P.I. SHPIRO (1983): Spectrum of the phase fluctuations at the ionosphere sounding, Izv. Vuzov. Radiofizika, 26 (7), 795-801.

Gershman, B.N., L.M. ERUKhIMOV and YU.YA.YASHIN (1984): Wave Phenomena in the Ionosphere and Space Plasma (Nauka, Moscow), pp. 392.

GDALEVICH, G.L., V.D. OZEROV, I.S. VSEKHSVYATSKAYA, L.N. NoviKova and T.N. SobolEVA (1980): Investigations of ionospheric variability on the height $500 \mathrm{~km}$ by the data of A.S.E. «Cosmoc 900», Geomagn. Aaeron., 20 (5), 809-816.

GibBons, G. (2002): Finding the GNSS Common Ground, Galileo's World, June 1.

Goodman, DJ.M. and J. AArons (1990): Ionospheric effects on modern electronic system, Proc. IEEE, 78 (3), 512-528.

GURTNER, W. (1993): RINEX: the Receiver Independent Exchange Format Version 2, http://igscb.jpl.nasa.gov/ igscb/data/format/rinex2.txt.

Hocke, K. and K. SCHLEGEL (1996): A review of atmospheric gravity waves and traveling ionospheric disturbances: 1982-1995, Ann. Geophysicae, 14 (5), 917-940.

HofMAnN-WellenhoF, B., H. LiChtenEGGer and J. Collins (1992): Global Positioning System: Theory and Practice (Springer-Verlag, New York), pp. 327.

Kaliev, M.Z., I.M. Krasnikov, U.G. Litvinov, B.D. CHEKANOv and A.F. YAKOVETS (1988): The spectrum fine structure of mediumscale ionospheric disturbances, Geomagn. Aaeron., 28 (2), 316-318.

Kharisov, V.N., A.I. Perov and V.A. Boldin (1998): The Global Satellite Radio Navigation GLONASS System (IPRZhR, Moscow), pp. 509 (in Russian).

KLOBUCHAR, J.A. (1986): Ionospheric time-delay algorithm for single-frequency GPS users, IEEE Trans. Aerosp. Electron. Syst., 23 (3), 325-331.

Kolosov, M.A., N.A. ARMAND and O.I. YAKovlev (1969): The Propagation of Radio Waves in Space Communication (Svyaz, Moscow), pp. 155.

Komrakov, G.P. and L.A. SkrebKova (1980): Study of parameters of ionospheric irregularities by the «Interkosmos-Kopernik 500» satellite, Ionos. Issled., 30, 49-52. 
Kravtsov, A.Yu., Z.I. FeIzUlin and A.G. Vinogradov (1983): The Propagation of Radio Waves Through the Earth's Ionosphere (Radio i Svyaz, Moscow), pp. 224.

Livingston, R.C., C.L. Rino, J.P. McCluRe and W.B. HANSON (1981): Spectral characteristics of medium-scale equatorial $F$ region irregularities, J. Geophys. Res., 86, 2421-2428.

MannuCCI, A.J., B.D. Wilson, D.N. Yuan, C.M. Ho, U.J. LINDQWISTER and T.F. RUNGE (1998): A global mapping technique for GPS-drived ionospheric TEC measurements, Radio Sci., 33, 565-582.

Oliver, W.L., Y. Otsuka, M. SATo, T. TAKAMI and S. FuKao (1997): A climatology of $F$ region gravity waves propagation over the middle and upper atmosphere radar, $J$. Geophys. Res., 102, 14449-14512.

Saito, A.M., M. Nishimura, M. Yamamoto, S. FuKao, M. Kubota, K. Shiokawa, Y. Otsuka, T. Tsugawa, T. OgaWa, M. IshiI, T. SAKANOI and S. MiYazaKi (2001): Traveling ionospheric disturbances detected in the FRONT campaign, Geophys. Res. Lett., 28, 689-692.

Saito, A., M. Nishimura, M. Yamamoto, S. FuKaO, T. TsugAWA, Y. OTSUKA, S. MiYAZAKI and M.C. Kelley (2002): Observations of traveling ionospheric disturbances and 3 -m scale irregularities in the nighttime $F$ region iono- sphere with the MU radar and a GPS network, Earth, Planets, Space, 54 (1), 31-44.

Schaer, S., W. GuRTNer and J. Feltens (1998): IONEX: the IONosphere map EXchange format version 1, in Proceeding of the IGS AC Workshop, edited by J.W. Dow, Darmstadt, Germany, February 9-11, 233-247.

SPOELSTRA, T.A. and H. KeLDER (1984): Effects produced by the ionosphere on radio interferometry, Radio Sci., 19, 779-788.

Thompson, R., J. Moran and Du. Swenson (1986): Interferometry and Synthesis in Radio Astronomy (John Wiley and Sons Inc., New York), pp. 556.

WiLson, B.D., A.J. ManNUCCI and C.D. Edwards (1995): Subdaily Northern Hemisphere maps using the IGS GPS network, Radio Sci., 30, 639-648.

YAKOVLeV, O.I. (1985): The Propagation of Radio Waves in Space (Nauka, Moscow), pp. 214

YAKUBOV, V.P. (1997): Doppler Very-Long-Baseline Interferometry (Vodolei, Tomsk), pp. 246.

(received February 10, 2003;

accepted September 10, 2003) 